\title{
Fatores relacionados à detecção de displasia em pacientes com esôfago de Barrett longo do Hospital Universitário Walter Cantídio
}

\section{Factors related to dysplasia detection in patients with long-segment Barrett's esophagus in the Walter Cantídio University Hospital}

\begin{abstract}
Nikaelle Ximenes Rios ${ }^{1}$. Danilo Frota Guimarães ${ }^{1}$. Isabele de Sá Silveira Melo². Cynthia Aben Athar Ponte ${ }^{3}$. Luciano Monteiro Franco ${ }^{4}$. Miguel Ângelo Nobre e Souza ${ }^{5}$. Marcellus Henrique Loiola Ponte de Souza ${ }^{6}$.

1 Médico(a), residente do Programa de Endoscopia Digestiva, Universidade Federal do Ceará (UFC), Fortaleza, Ceará, Brasil. 2 Médica Gastroenterologista, Hospital Universitário Walter Cantídeo (HUWC), Universidade Federal do Ceará (UFC), Empresa Brasileira de Serviços Hospitalares (EBSERH), Fortaleza, Ceará, Brasil. 3 Doutora em Ciências Médicas, Médica Endoscopista pelo Hospital Universitário Walter Cantídeo (HUWC), Universidade Federal do Ceará (UFC), Empresa Brasileira de Serviços Hospitalares (EBSERH), Fortaleza, Ceará, Brasil. 4 Médico Patologista, preceptor do Programa de Patologia pela Universidade Federal do Ceará(UFC), Fortaleza, Ceará, Brasil. 5 Professor Adjunto, Universidade Federal do Ceará (UFC), chefe do Serviço de Endoscopia Digestiva do Hospital Universitário Walter Cantídio (HUWC), Fortaleza, Ceará, Brasil. 6 Professor Associado, Universidade Federal do Ceará (UFC), Supervisor da Residência Médica em Endoscopia Digestiva, Hospital Universitário Walter Cantídio (HUWC), Fortaleza, Ceará, Brasil.
\end{abstract}

\section{RESUMO}

Introdução: pacientes com doença do refluxo gastroesofágico (DRGE) têm de 10 a 15\% de chance de desenvolver esôfago de Barrett. Este pode evoluir para displasia e adenocarcinoma esofágico. Objetivo: avaliar fatores relacionados à deteç̧ão de displasia em pacientes com esôfago de Barrett longo. Metodologia: estudo retrospectivo com análise de informações de 18 pacientes com diagnóstico de esôfago de Barrett longo, colhidas de bancos de dados dos serviços de Endoscopia e Patologia e prontuários do Hospital Universitário Walter Cantídio (HUWC-UFC), no período de 2010 a 2015. Os pacientes foram divididos em dois grupos para comparação, grupo sem displasia $(\mathrm{N}=11)$ e grupo com displasia/adenocarcinoma $(\mathrm{N}=7)$. Resultados: do total de 18 pacientes com esôfago de Barrett longo no HUWC, 72\% eram do sexo masculino, 47\% tinham mais de 50 anos, $64 \%$ apresentavam sintomas típicos de DRGE, 23\% eram tabagistas, 77\% tinha hérnia hiatal, 50\% realizaram fundoplicatura. Não houve diferenças estatísticas destas características entre o grupo com e sem displasia. Entretanto, o grupo com displasia teve uma média de 5 endoscopias/paciente, enquanto o grupo sem displasia teve apenas 2 endoscopias/paciente $(\mathrm{p}<0,05)$. Conclusão: $\mathrm{O}$ maior número de endoscopias realizadas foi um fator relacionado à maior detecção de displasia/adenocarcinoma em pacientes com esôfago de Barrett no HUWC-UFC.

Palavras-chave: Esôfago de Barrett. Endoscopia. Neoplasias esofágicas.

\section{ABSTRACT}

Introduction: Patients with gastroesophageal reflux disease (GERD) have a 10-15\% chance of developing Barrett's esophagus. This one may progress to dysplasia and esophageal adenocarcinoma. Objective: To evaluate factors related to the detection of dysplasia in patients with long-segment Barrett's esophagus. Methods: Retrospective study with information analysis of 18 patients diagnosed with long-segment Barrett's esophagus, collected from Endoscopy and Pathology databases and medical records of the Hospital Universitário Walter Cantídio (HUWC-UFC), from 2010 to 2015. Patients were divided into two groups for comparison, group without dysplasia $(\mathrm{N}=11)$ and group with dysplasia/adenocarcinoma $(\mathrm{N}=7)$. Results: Of the 18 patients with long-segment Barrett's esophagus in HUWC, $72 \%$ were male, $47 \%$ were older than 50 years, $64 \%$ had typical symptoms of GERD, $23 \%$ were smokers, $77 \%$ had hiatal hernia, $50 \%$ performed fundoplication. There were no statistical differences of these characteristics between the group with and without dysplasia. However, the group with dysplasia had an average of 5 endoscopies/patient, whereas the group without dysplasia had only 2 endoscopies/patient $(\mathrm{p}<0.05)$. Conclusion: The largest number of endoscopies performed was a factor related to the greater detection of dysplasia/adenocarcinoma in patients with Barrett's esophagus in HUWC-UFC.

Keywords: Barrett esophagus. Endoscopy. Esophagealneoplasms.

Autor correspondente: Marcellus Henrique Loiola Ponte de Souza, Rua Coronel Nunes de Melo, 1315, Rodolfo Teófilo, Fortaleza, Ceará. CEP: 60430-270. Telefone: +55 85 3366-8167. E-mail: souzamar@ufc.br

Conflito de interesses: Não há qualquer conflito de interesses por parte de qualquer um dos autores.

Recebido em: 18 Mar 2017; Revisado em: 09 Jun 2017; Aceito em: 24 Set 2017. 


\section{INTRODUÇÃO}

Estudos populacionais recentes sugerem que a doença do refluxo gastroesofágico (DRGE) tem aumentado em prevalência ao redor do mundo. Pacientes com diagnóstico de DRGE têm chance de 10-15\% de desenvolver esôfago de Barrett, uma transformação do epitélio escamoso colunar no esôfago distal em metaplasia intestinal, aumentando o risco de desenvolvimento de adenocarcinoma esofágico. ${ }^{1}$

Os principais fatores de risco para o desenvolvimento de esôfago de Barrett são sintomas crônicos de DRGE $(>5$ anos), ${ }^{2}$ sexo masculino, ${ }^{3}$ idade avançada ( $>50$ anos $),{ }^{4}$ uso de tabaco, ${ }^{5}$ obesidade central, ${ }^{6}$ raça caucasiana e parentes de primeiro grau com o diagnóstico. ${ }^{7}$ A suspeita de esôfago de Barrett é feita através da realização de endoscopia digestiva alta (EDA), com a visualização do epitélio vermelho-salmão que se estende proximalmente, a partir de $1 \mathrm{~cm}$ acima da linha Z. O diagnóstico é dado através da realização de biópsias dessa área, com o achado de metaplasia intestinal no resultado anatomopatológico.

O esôfago de Barrett pode ser classificado em curto, quando apresenta menos de $3 \mathrm{~cm}$ de extensão e longo, quando se estende por $3 \mathrm{~cm}$ ou mais, proximalmente. ${ }^{8}$ Um fator de risco importante para o desenvolvimento de neoplasia esofágica é a extensão do esôfago de Barrett. ${ }^{9}$ Outros fatores relacionados são idade avançada, obesidade central e uso de tabaco. ${ }^{1} \mathrm{O}$ uso de medicações como inibidor da bomba de prótons (IBP), anti-inflamatórios não-esteroidais e estatinas protegem o epitélio esofágico metaplásico de evoluir para adenocarcinoma. ${ }^{10}$

A partir da metaplasia intestinal do esôfago de Barrett, pode haver transformação para displasia de baixo grau, displasia de alto grau e adenocarcinoma esofágico. Essa progressão é estimada em menos de $0,5 \%$ ao ano, com aumento para $0,7 \%$ ao ano para displasia de baixo grau e $7 \%$ ao ano para displasia de alto grau. ${ }^{11,12} \mathrm{Com}$ isso, a vigilância endoscópica se faz necessária, com a realização de biópsias seriadas. ${ }^{13}$

Neste estudo, buscou-se descrever características clínicas, endoscópicas e anatomopatológicas de pacientes com esôfago de Barrett longo, no serviço de Endoscopia do Hospital Universitário Walter Cantídio (HUWC), bem como avaliar os fatores envolvidos na detecção de displasia.

\section{MATERIAIS E MÉTODOS}

Estudo retrospectivo com análise de informações colhidas de bancos de dados dos serviços de Endoscopia e Patologia, além de prontuários do Hospital Universitário Walter Cantídio da Universidade Federal do Ceará (UFC), no período de maio de 2010 a agosto de 2015. Foram selecionados pacientes que receberam o diagnóstico endoscópico de esôfago de Barrett longo, submetidos a biópsias aleatórias do epitélio vermelho-salmão esofágico visto durante o exame, e confirmado pela realização de anatomopatológico. Patologistas experientes avaliaram e confirmaram a metaplasia intestinal e o grau de displasia, quando presente, nas amostras. Foram avaliadas as características clínicas, endoscópicas e anatomopatológicas através de um formulário estruturado. O programa estatístico GraphPad Prisma e o teste de Fisher foram utilizados para avaliação estatística. Este artigo foi aprovado pelo comitê de ética em pesquisa em seres humanos do Hospital Universitário Walter Cantídio (Protocolo: 45868215.7.0000.5045).

Foi realizada a análise comparativa entre pacientes sem displasia com aqueles que apresentavam displasia/ adenocarcinoma esofágico quanto aos fatores de risco para esôfago de Barrett, como: sexo masculino, idade $>50$ anos, sintomas típicos de DRGE, tabagismo, além de características endoscópicas como: hernia hiatal, seu tamanho, fundoplicatura e seu número, uso de inibidor da bomba de prótons, sintomas típicos de DRGE na última consulta, além do número de endoscopias realizadas por cada grupo.

\section{RESULTADOS}

\section{Pacientes}

No período de maio de 2010 a agosto de 2015, foram realizadas 16.230 endoscopias digestivas altas no Setor de Endoscopia do Hospital Universitário Walter Cantídio (HUWC). Desses exames, 398 tinham como laudo esôfago de Barrett, correspondendo a 156 pacientes. Desses pacientes, 134 receberam o diagnóstico endoscópico de esôfago de Barrett curto (extensão $<3 \mathrm{~cm}$ ) e 22 pacientes de esôfago de Barrett longo (extensão $\geq 3 \mathrm{~cm}$ ). Desses pacientes, em 4 não foi confirmada a presença de metaplasia intestinal no exame anatomopatológico. Assim, 18 pacientes preencheram critério de inclusão para avaliação de fatores associados ao risco de esôfago de Barrett e desenvolvimento de displasia.

A fim de avaliar os fatores relacionados à presença e à detecção da displasia neste grupo, os pacientes foram divididos em dois grupos para comparação. Um grupo de 11 pacientes sem displasia e um grupo de 7 pacientes com displasia/adenocarcinoma esofágico no resultado do histopatológico.

\section{Características epidemiológicas}

Dos 18 pacientes incluídos na pesquisa, $72 \%$ eram do sexo masculino, $47 \%$ tinham idade $>50$ anos, $64 \%$ apresentavam sintomas típicos de refluxo gastroesofágico (DRGE), 23\% tinham história de tabagismo (Tabela 1).

\section{Características endoscópicas}

Dos pacientes com esôfago de Barrett longo, 77\% tinham hérnia hiatal por deslizamento. Desses, $66 \%$ com hérnia hiatal de pequeno porte ( 2 a $4 \mathrm{~cm}$ ), e $11 \%$ de médio a grande porte $(>4 \mathrm{~cm})$. Metade desses pacientes com hérnia já haviam sido submetidos à fundoplicatura como forma de controle da DRGE, sendo 33\% realizada uma vez e 17\% duas vezes. Destes pacientes, $33 \%$ apresentavam a fundoplicatura desfeita na última endoscopia realizada (Tabela 1). 
Tabela 1. Características dos pacientes com esôfago de Barrett longo do Hospital Universitário Walter Cantídio.

\begin{tabular}{|c|c|}
\hline Características & Porcentagem $(\%)$ \\
\hline Sexo Masculino & $72 \%$ \\
\hline Idade $>50$ anos & $47 \%$ \\
\hline Sintomas típicos de DRGE & $64 \%$ \\
\hline Tabagismo & $23 \%$ \\
\hline Hérnia hiatal & $77 \%$ \\
\hline Hérnia hiatal (2-4cm) & $66 \%$ \\
\hline Hérnia hiatal $(>4 \mathrm{~cm})$ & $11 \%$ \\
\hline Fundoplicatura & $50 \%$ \\
\hline Uso de IBP na última consulta & $41 \%$ \\
\hline Sintomas típicos de DRGE na última consulta & $29 \%$ \\
\hline
\end{tabular}

\section{Outros dados}

Dos pacientes com esôfago de Barrett longo, $41 \%$ faziam uso de inibidor da bomba de prótons na última consulta e $29 \%$ mantinham sintomas típicos de DRGE (Tabela 1). Cada paciente foi submetido a uma média de 3 endoscopias. Somente um paciente realizou pHmetria para avaliação de controle de DRGE.

\section{Análise comparativa entre pacientes sem displasia e com displasia/adenocarcinoma esofágico}

Dos pacientes sem displasia, $72 \%$ eram do sexo masculino, $45 \%$ maiores de 50 anos, $63 \%$ apresentavam sintomas típicos de DRGE, $9 \%$ eram tabagistas e $81 \%$ já haviam tido hérnia hiatal. Desses, $63 \%$ com hérnia de pequeno porte ( 2 a $4 \mathrm{~cm})$, e $18 \%$ de médio a grande porte $(>4 \mathrm{~cm}) .45 \%$ desses pacientes já haviam sido submetidos à fundoplicatura, sendo $27 \%$ uma vez e $18 \%$ duas vezes. $40 \%$ apresentavam a fundoplicatura desfeita (Tabela 2). A média do número de endoscopias realizadas por esse grupo foi de 2 endoscopias/paciente.
Dos pacientes que apresentavam displasia/adenocarcinoma esofágico, $71 \%$ eram do sexo masculino, $66 \%$ maiores de 50 anos, $66 \%$ apresentavam sintomas típicos de DRGE e metade tinha história de tabagismo, $71 \%$ tinham hérnia hiatal, sendo todas de pequeno porte $(2 \mathrm{a} 4 \mathrm{~cm})$ e $57 \%$ dos pacientes com hérnia hiatal já haviam sido submetidos à fundoplicatura (Tabela 2). A média do número de endoscopias realizadas por esse grupo foi de 5 endoscopias/paciente.

$\mathrm{Na}$ análise comparativa dos dados epidemiológicos (Tabela 2), não houve diferença significativa $(\mathrm{p}>0,05)$ entre os grupos sem displasia e com displasia/adenocarcinoma, porém, os pacientes com displasia/adenocarcinoma apresentaram maior frequência de tabagismo e de idade superior a 50 anos.

A Figura 1 mostra que o número de endoscopias realizadas pelo grupo com displasia/adenocarcinoma esofágico foi significativamente maior que o grupo sem displasia $(\mathrm{p}<0,05)$.

Tabela 2. Comparação das características dos pacientes com esôfago de Barrett longo sem displasia com aqueles com displasia/adenocarcinoma esofágico.

\begin{tabular}{llll}
\hline Características & Sem displasia & $\begin{array}{l}\text { Com displasia/ } \\
\text { adenocarcinoma }\end{array}$ & $\mathrm{p}$ \\
\hline Sexo masculino & $72 \%$ & $71 \%$ & $>0,05$ \\
Idade $>50$ anos & $45 \%$ & $66 \%$ & $>0,05$ \\
Sintomas típicos de DRGE & $63 \%$ & $66 \%$ & $>0,05$ \\
Tabagismo & $9 \%$ & $50 \%$ & $>0,05$ \\
Hérnia hiatal & $81 \%$ & $71 \%$ & $>0,05$ \\
Hérnia hiatal $(2-4 \mathrm{~cm})$ & $63 \%$ & $71 \%$ & $>0,05$ \\
Hérnia hiatal $(>4 \mathrm{~cm})$ & $18 \%$ & $0 \%$ & $>0,05$ \\
Fundoplicatura & $45 \%$ & $57 \%$ & $>0,05$ \\
Uso de IBP na última consulta & $45 \%$ & $33 \%$ & $>0,05$ \\
\hline
\end{tabular}

*Teste de Fisher 
Figura 1. Comparação do número de endoscopias realizadas pelos pacientes com esôfago de Barrett longo sem displasia com aqueles com displasia/adenocarcinoma. ${ }^{*} \mathrm{p}<0,05$ teste de Mann-Whitney.

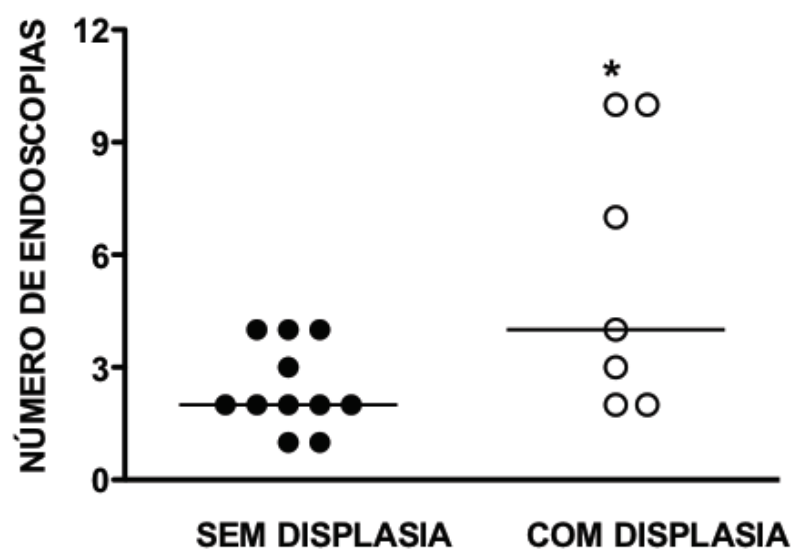

\section{DISCUSSÃO}

Pacientes com doença do refluxo gastroesofágico têm $10 \%$ de chance de desenvolver esôfago de Barrett. Esta alteração pode levar à displasia e evoluir para adenocarcinoma esofágico, numa incidência de 0,5 a $7 \%$ ao ano, dependendo da presença e do grau de displasia. Fatores de risco para o desenvolvimento de esôfago de Barrett e de displasia foram avaliados no nosso estudo, com atenção aos fatores relacionados à detecção de displasia nos pacientes do HUWC. ${ }^{14}$

A maioria dos pacientes era do sexo masculino, tinha sintomas crônicos de DRGE e possuía hérnia hiatal, fatores relacionados ao desenvolvimento de esôfago de Barrett. Asanuma et al, não encontraram um fator que causasse maior desenvolvimento de esôfago de Barrett, mas percebeu que na verdade as mulheres em idade reprodutiva teriam o efeito protetor anti-inflamatório do estrogênio, levando a uma maior resistência epitelial, atrasando o desenvolvimento de adenocarcinoma esofágico. ${ }^{15}$ De acordo com Roman $\mathrm{S}$ et al, a presença de hérnia hiatal, por deslizamento principalmente, é caracterizada por uma separação entre o esfíncter esofágico inferior e o diafragma crural, que normalmente trabalham em sinergia para aumentar a barreira antirrefluxo. Assim, a hérnia de hiato altera profundamente o processo normal de depuração de ácido, permitindo o "refluxo" do suco gástrico para o esôfago distal durante o relaxamento do esfíncter esofagiano inferior induzido pela deglutição, circunstância normalmente impedida pela contração crural do diafragma. ${ }^{16}$

Avaliando fatores para desenvolvimento de displasia e adenocarcinoma esofágico, observou-se que a maioria dos

\section{REFERÊNCIAS}

1. Shaheen NJ, Falk GW, Iyer PG, Gerson LB; American College of Gastroenterology. ACG clinical guideline: diagnosis and management of Barrett's Esophagus. Am J Gastroenterol. 2016;111(1):30-50.

2. Lieberman DA, Oehlke M, Helfand M. Risk factors for Barrett's pacientes do grupo com essas alterações tinha idade maior que 50 anos. Em um estudo recente, percebeu-se que a tendência de desenvolvimento de displasia de alto grau e adenocarcinoma aumenta com a duração da vigilância, mas de forma mais marcante, há uma relação maior entre a idade avançada e a taxa de detecção destas alterações. ${ }^{17}$

Metade dos pacientes com displasia/adenocarcinoma esofágico tinha história de tabagismo, em uma proporção bem maior neste grupo. Fato percebido em nosso trabalho e comprovado em estudos que demonstram que o cigarro induz alterações na metilação do DNA das células da mucosa esofágica, levando ao desenvolvimento de adenocarcinoma em esôfago de Barrett. ${ }^{18}$

A maioria dos pacientes do grupo com displasia estava sem uso de inibidores da bomba de prótons. O uso desses medicamentos e o risco de desenvolvimento de adenocarcinoma esofágico têm sido conflitantes. Uma revisão sistemática recente com metanálise de vários estudos investigou a associação entre o uso de medicamentos supressores de ácidos e adenocarcinoma esofágico no esôfago de Barrett. Foi observado que o uso de inibidor da bomba de prótons foi associado a uma redução de risco de $71 \%$ na progressão para adenocarcinoma esofágico e/ ou displasia de alto grau em um uso dependente da duração de mais do que 2-3 anos após o diagnóstico de esôfago de Barrett. ${ }^{19}$ Este fato alerta para um cuidado na manutenção do uso de IBP em pacientes com esôfago de Barrett.

A média de endoscopias realizadas por pacientes com displasia foi bem maior que no grupo sem displasia. Esse maior número de endoscopias realizadas foi um fator relacionado à maior detecção de displasia/adenocarcinoma em pacientes com esôfago de Barrett no HUWC-UFC. Dado observado também em estudos populacionais, como uma metanálise recente de 24 estudos de coorte em adultos com esôfago de Barrett, demonstrando que $25 \%$ do diagnóstico de adenocarcinoma esofágico é feito somente após a repetição da endoscopia digestiva em um ano do diagnóstico do esôfago de Barrett. ${ }^{20}$ Muitas vezes as biópsias esofágicas realizadas no momento da endoscopia não detectam displasia/adenocarcinoma por erro de amostragem, por presença de esofagite erosiva, pelo não seguimento do protocolo de biópsias ou pelo uso de inibidor da bomba de prótons. $^{21}$ A maior repetição da endoscopia permite o diagnóstico precoce de displasia e adenocarcinoma esofágico, que pode ter sido perdido em exame anterior, levando a uma redução da mortalidade desses doentes.

Podemos concluir que o maior número de endoscopias realizadas foi um fator relacionado à maior detecção de displasia/adenocarcinoma esofágico em pacientes com esôfago de Barrett. esophagus in community-based practice. Am J Gastroenterol. 1997;92(8):1293-7.

3. Rubenstein JH, Scheiman JM, Sadeghi S, Whiteman D, Inadomi JM. Esophageal adenocarcinoma incidence in individuals with 
gastroesophageal reflux: synthesis and estimates from population studies. Am J Gastroenterol. 2011;106(2):254-60.

4. Rubenstein JH, Mattek N, Eisen G. Age-and sex-specific yield of Barrett's esophagus by endoscopy indication. Gastrointest Endosc. 2010;71(1):21-7.

5. Andrici J, Cox MR, Eslick GD. Cigarette smoking and the risk of Barrett's esophagus: a systematic review and meta-analysis. J Gastroenterol Hepatol. 2013;28(8):1258-73.

6. Singh S, Sharma AN, Murad MH, Buttar NS, El-Serag HB, Katzka DA, et al. Central adiposity is associated with increased risk of esophageal inflammation, metaplasia, and adenocarcinoma: a systematic review and meta-analysis. Clin Gastroenterol Hepatol. 2013;11(11):1399-412.

7. Chak A, Lee T, Kinnard MF, Brock W, Faulx A, Willis J, et al. Familial aggregation of Barrett's oesophagus, oesophageal adenocarcinoma, and oesophagogastric junctional adenocarcinoma in Caucasian adults. Gut. 2002;51(3):323-8.

8. Sharma P, Morales TG, Sampliner RE. Short segment Barrett's esophagus-the need for standardization of the definition and of endoscopic criteria. Am J Gastroenterol. 1998;93(7):1033-6.

9. Gopal DV, Lieberman DA, Magaret N, Fennerty MB, Sampliner RE, Garewal HS, et al. Risk factors for dysplasia in patients with Barrett's esophagus (BE): results from a multicenter consortium. Dig Dis Sci. 2003;48(8):1537-41.

10. Zhang S, Zhang XQ, Ding XW, Yang RK, Huang SL, Kastelein $\mathrm{F}$, et al. Cyclooxygenase inhibitors use is associated with reduced risk of esophageal adenocarcinoma in patients with Barrett's esophagus: a meta-analysis. Br J Cancer. 2014;110(9):2378-88.

11. Rastogi A, Puli S, El-Serag HB, Bansal A, Wani S, Sharma P. Incidence of esophageal adenocarcinoma in patients with Barrett's esophagus and high-grade dysplasia: a meta-analysis. Gastrointest Endosc. 2008;67(3):394-8.

12. Shaheen NJ, Sharma P, Overholt BF, Wolfsen HC, Sampliner RE, Wang KK, et al. Radiofrequency ablation in Barrett's esophagus with dysplasia. N Engl J Med. 2009;360:2277-88.
13. Harrison R, Perry I, Haddadin W, McDonald S, Bryan R, Abrams $\mathrm{K}$, et al. Detection of intestinal metaplasia in Barrett's esophagus: an observational comparator study suggests the need for a minimum of eight biopsies. Am J Gastroenterol. 2007;102(6):1154- 61 .

14. Spechler SJ. Barrett esophagus and risk of esophageal cancer: a clinical review. JAMA. 2013;310(6):627-36.

15. Asanuma K, Iijima K, Shimosegawa T. Gender difference in gastro-esophageal reflux diseases. World $\mathrm{J}$ Gastroenterol. 2016;22(5):1800-10.

16. Roman S, Kahrilas PJ. Mechanisms of Barrett's esophagus (clinical): LES dysfunction, hiatal hernia, peristaltic defects. Best pract Res Clin Gastroenterol. 2015;29(1):17-28.

17. Gatenby P, Bhattacharjee S, Wall C, Caygill C, Watson A. Risk stratification for malignant progression in Barrett's esophagus: gender, age, duration and year of surveillance. World J Gastroenterol. 2016;22(48):10592-600.

18. Kaz AM, Wong C, Varadan V, Willis JE, Chak A, Grady WM. Global DNA methylation patterns in Barrett's esophagus, dysplastic Barrett's, and esophageal adenocarcinoma are associated with BMI, gender, and tobacco use. Clin Epigenetics. 2016;8:111.

19. Singh S, Garg SK, Singh PP, Iyer PG, El-Serag HB. Acidsuppressive medications and risk of esophageal adenocarcinoma in patients with Barrett's esophagus: a systematic review and metaanalysis. Gut. 2014;63(8):1229-37.

20. Visrodia K, Singh S, Krishnamoorthi R, Ahlquist DA, Wang KK, Iyer PG, et al. Magnitude of missed esophageal adenocarcinoma after Barrett's esophagus diagnosis: a systematic review and metaanalysis. Gastroenterology. 2016;150(3):599-607.

21. Visrodia K, Iyer PG, Schleck CD, Zinsmeister AR, Katzka DA. Yield of repeat endoscopy in Barrett's esophagus with no dysplasia and low-grade dysplasia: a population-based study. Dig Dis Sci. 2016;61(1):158-67.

\section{Como citar:}

Rios NX, Guimarães DF, Melo IS, Ponte CA, Franco LM, Souza MA, et al. Fatores relacionados à detecção de displasia em pacientes com esôfago de Barrett longo do Hospital Universitário Walter Cantídio. Rev Med UFC. 2018 abr-jun;58(2):36-40. 